Case Report

\title{
Triple Synchronous Primary Neoplasms of the Cervix, Endometrium, and Ovary: A Rare Case Report and Summary of All the English PubMed-Indexed Literature
}

\author{
Ahmed Abu-Zaid, ${ }^{1,2}$ Mohannad Alsabban, ${ }^{3}$ Mohammed Abuzaid, ${ }^{4}$ Osama Alomar, ${ }^{3}$ \\ Hany Salem, ${ }^{3}$ and Ismail A. Al-Badawi ${ }^{1,3}$ \\ ${ }^{1}$ College of Medicine, Alfaisal University, Riyadh, Saudi Arabia \\ ${ }^{2}$ College of Graduate Health Sciences, University of Tennessee Health Science Center, Memphis, TN, USA \\ ${ }^{3}$ Department of Obstetrics \& Gynecology, King Faisal Specialist Hospital \& Research Centre, Riyadh, Saudi Arabia \\ ${ }^{4}$ Department of Obstetrics \& Gynecology, King Fahad Medical City, Riyadh, Saudi Arabia \\ Correspondence should be addressed to Ahmed Abu-Zaid; aabuzaid@live.com
}

Received 12 March 2017; Accepted 26 July 2017; Published 23 August 2017

Academic Editor: Maria Grazia Porpora

Copyright (C) 2017 Ahmed Abu-Zaid et al. This is an open access article distributed under the Creative Commons Attribution License, which permits unrestricted use, distribution, and reproduction in any medium, provided the original work is properly cited.

\begin{abstract}
The incidence rate of triple or more synchronous primary neoplasms of the female genital system is exceedingly uncommon. To the best of our knowledge, only 13 such cases have been reported in the PubMed-indexed English literature. Herein, we report a single case of triple synchronous primary neoplasms of the cervix, endometrium, and left ovary with three distinct histological patterns that were not reported previously. Moreover, we briefly present a summary table of all the English PubMed-indexed cases of triple or more synchronous primary neoplasms of the female genital system $(n=13)$.
\end{abstract}

\section{Introduction}

Synchronous primary neoplasms are defined when two or more neoplasms take place concurrently in the same patient. These neoplasms should be histologically discrete and separated from each other by means of healthy tissues, such as basal lamina or stroma [1]. Double gynecological neoplasms are occasionally observed, and the most commonly reported combination is endometrial-ovarian neoplasms $[2,3]$. The incidence of triple or more synchronous primary neoplasms of the female genital system is exceedingly uncommon. To the best of our knowledge, only 13 such cases have been reported in the PubMed-indexed English literature [1, 2, 414]. Herein, we report a single case of triple synchronous primary neoplasms of the cervix, endometrium, and right ovary with three distinct histological patterns that were not reported previously. Moreover, we briefly present a summary table of all English PubMed-indexed existing cases of triple or more synchronous primary neoplasms of the female genital system $(n=13)$.

\section{Case Report}

A 55-year-old multiparous woman was referred to our hospital as a case of pelvic/abdominal mass for 2 months. The mass was associated with progressive abdominal distention, left lower abdominal pain, and occasional vaginal bleeding. Her past medical and surgical histories were unremarkable. Upon pelvic examination, a cystic, mobile, nontender pelvic mass was palpated up to the umbilicus. There were no abnormal cervical growths. Laboratory findings showed a slightly elevated CA-125 level of $36.6 \mathrm{U} / \mathrm{mL}$ (normal range: $0-35 \mathrm{U} / \mathrm{mL}$ ).

Two imaging studies were conducted: ultrasound (US) and computed tomography (CT) scan. US showed a $15 \mathrm{~cm}$ pelvic mass at the left adnexal site. The mass was cystic and contained solid areas. CT scan showed a heterogeneous $14 \times$ $12 \mathrm{~cm}$ pelvic/abdominal mass with solid components, along with multiple prominent left pelvic and para-aortic lymph nodes that were highly suspicious for metastasis. The mass was seen between uterus and rectum. The mass was most 


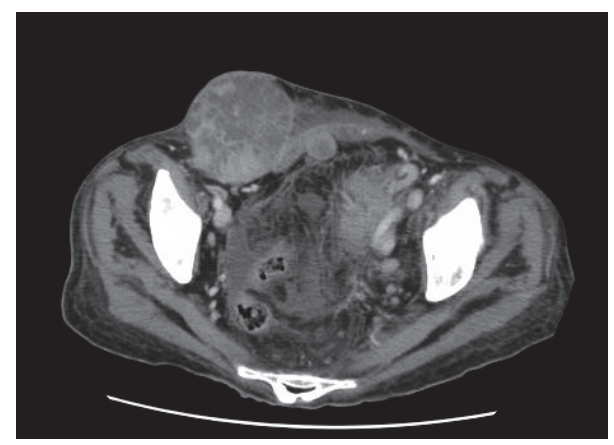

Figure 1: Axial CT scan showing heterogeneous $14 \times 12 \mathrm{~cm}$ pelvicabdominal mass between uterus and rectum, most likely originating from the left ovary and inseparable from the posterior uterus.

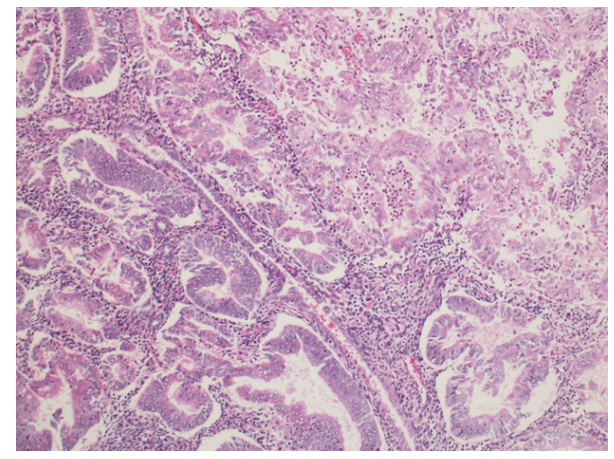

FIgURE 2: Histopathological examination showing grade-I endometrioid adenocarcinoma of the uterus (H\&E stain).

likely originating from the left ovary and inseparable from the posterior uterus (Figure 1). In view of an underlying neoplastic process, a surgical staging operation was planned.

The surgical staging operation consisted of total abdominal hysterectomy, bilateral salpingo-oophorectomy, infracolic omentectomy, sampling from the pelvic lymph nodes and para-aortic lymph nodes, and multiple biopsy specimens from various peritoneal sites. During the laparotomy, the pelvic mass was found to be most likely originating from the left ovary. The uterus was bulky and the right ovary was grossly normal. There were no ascites or other gross intraabdominal lesions. All resected specimens were examined for histopathological analysis.

Histopathological examination showed grade-I endometrioid adenocarcinoma of the uterus with no lymphovascular invasion and less than 50\% invasion into the myometrium (Figure 2). Both ovaries were examined, and the microscopic appearance of the left ovary revealed clear-cell carcinoma with no lymphovascular invasion (Figure 3 ). The right ovary and bilateral fallopian tubes were normal. Microscopic examination of the cervix exhibited poorly differentiated squamous cell carcinoma (Figure 4). The omentum and bilateral pelvic and para-aortic lymph nodes were negative for metastasis.

Therefore, the final histopathological diagnosis was triple synchronous primary stage $1 \mathrm{~B} 1$ poorly differentiated squamous cell carcinoma of the cervix (pTNM: T1B1 Nx M0),

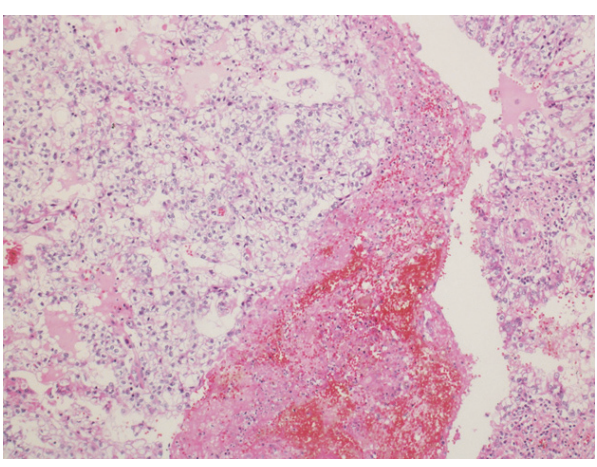

FIGURE 3: Histopathological examination showing clear-cell carcinoma of left ovary (H\&E stain).

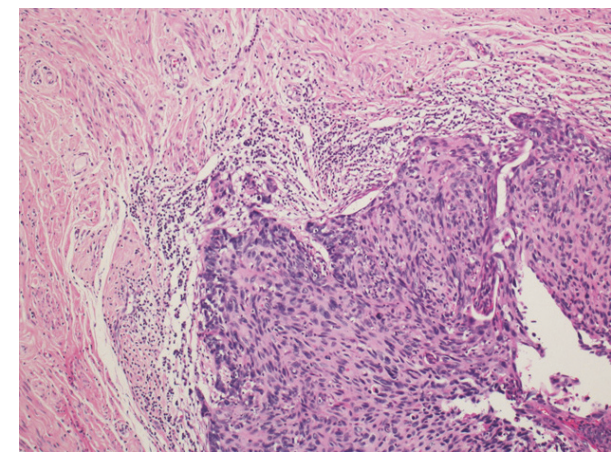

FIgURE 4: Histopathological examination showing poorly differentiated squamous cell carcinoma of the cervix (H\&E stain).

stage 1A grade-I endometrioid adenocarcinoma of the uterus (pTNM: T1A Nx M0), and stage 1A clear-cell carcinoma of the left ovary (pTNM: T1A Nx M0).

The case was discussed in the multidisciplinary tumor board meeting and the recommendation was to start adjuvant therapy. The adjuvant therapy consisted of chemotherapy and radiation therapy. The adjuvant chemotherapy was primarily intended for the high-risk ovarian cancer (clear-cell carcinoma) and included six cycles of paclitaxel $175 \mathrm{mg} / \mathrm{m}^{2}$ plus carboplatin (area under the curve [AUC] 6). The adjuvant radiation therapy was primarily intended for the high-risk cervical cancer (poorly differentiated squamous cell carcinoma) and consisted of external beam radiation therapy (EBRT) of 51 Gy delivered in 26 fractions.

The management plan was discussed with the patient; however, she refused the adjuvant treatment. Three months later, she presented to clinic with radiological evidence of recurrence, as follows: large local recurrence in the left iliac fossa, multiple metastatic masses in the abdominal and pelvic cavities, and multiple metastatic retroperitoneal and pelvic lymph nodes.

\section{Discussion}

The incidence rate of double synchronous primary gynecological neoplasms is relatively uncommon and ranges from $0.6 \%$ to $5.4 \%[2,3,15-17]$. The incidence rate of triple or more 


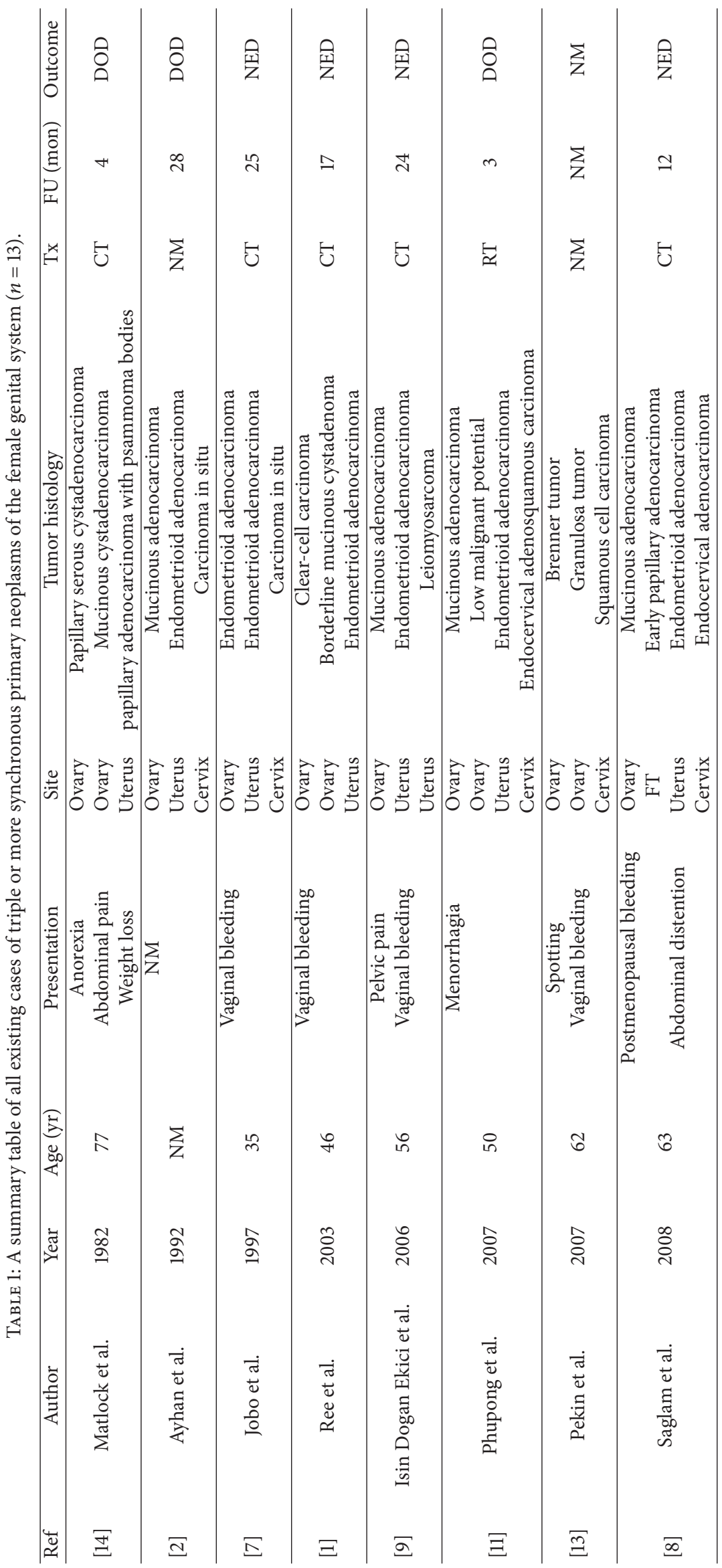




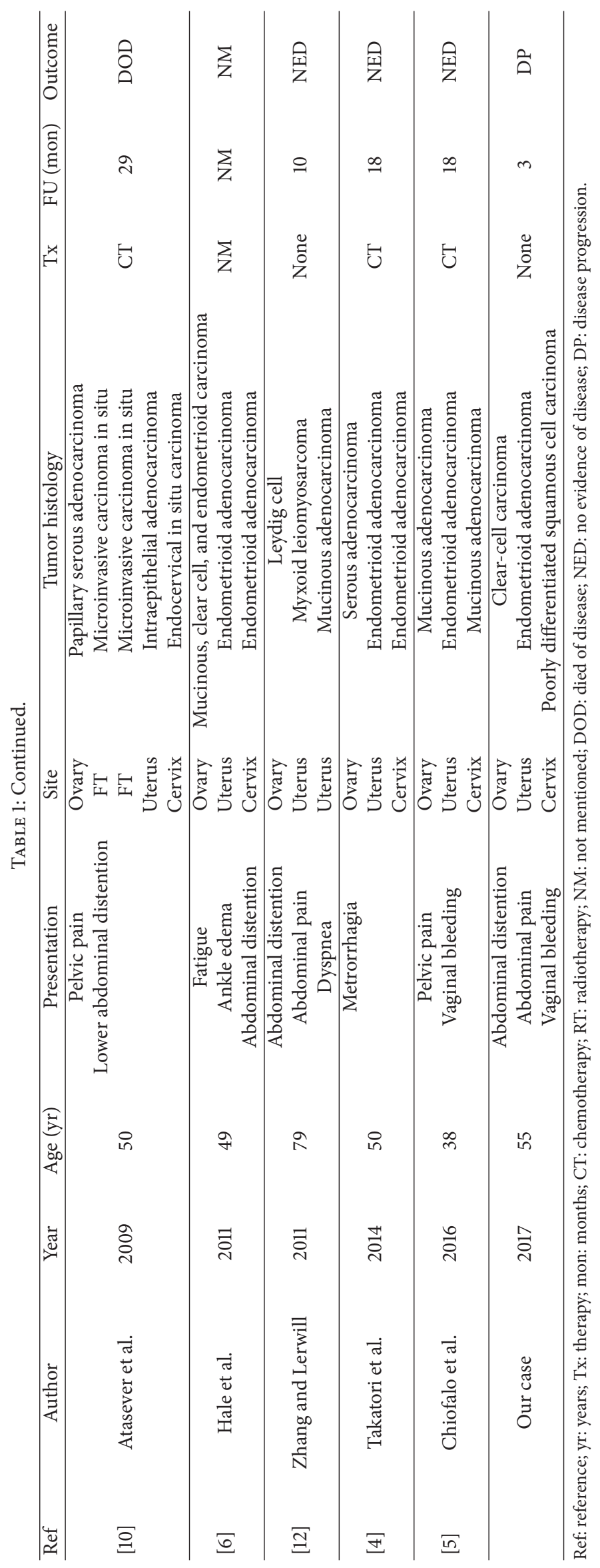


synchronous primary neoplasms of the female genital system is exceedingly uncommon. To the best of our knowledge, only 13 such cases have been reported in the PubMed-indexed English literature [1, 2, 4-14] (Table 1). There was only one case of quintuple synchronous neoplasms [10] and two cases of quadruple synchronous neoplasms $[8,11]$. On the other hand, there were only 10 cases of triple synchronous neoplasms. In our study, the distinctive combination of poorly differentiated squamous cell carcinoma of the cervix, gradeI endometrioid adenocarcinoma of the uterus and clearcell carcinoma of the left ovary has never been previously reported.

The etiology of synchronous primary neoplasms of the female genital system remains poorly defined. It has been assumed that in genetically predisposed individuals, the Mullerian tissues with similar embryological origin may respond as a single structural entity when simultaneously exposed to carcinogenic, hormonal, therapeutic, or other triggering factors [2]. In our study, there were three different histological subtypes identified in the surgical specimens (squamous, endometrioid, and clear-cell). This unusual presentation raises thoughts about potential underlying epigenetic/bimolecular explanations, and this is an interesting arena for future research.

Several clinicopathological criteria have been suggested to assist clinicians and pathologists in distinguishing synchronous primary gynecological neoplasms from related metastatic foci. These criteria include either one major criterion or all the four minor criteria. The one major criterion is the existence of distinct histological types of the neoplasms. The four minor criteria include (a) neoplasms which are limited to primary locations, (b) absence of direct extension between neoplasms, (c) absence of lymphovascular neoplastic invasion, and (d) absence of distant metastasis $[18,19]$. In our case, all the above-mentioned major and minor criteria were met, hence confirming the diagnosis of triple synchronous primary neoplasms of the female genital system.

It is critically crucial to differentiate between synchronous primary gynecological neoplasms and related metastatic diseases. This is because both management and prognosis vary substantially. Regarding prognosis, synchronous primary gynecological neoplasms are associated with better survival rates than metastatic or advanced primary ones [2, 3, 17]. This observation may be attributable to the younger age of presentation, earlier disease stage, and lower disease grade at the time of clinical diagnosis [17]. The prognosis of a triple neoplasm is largely determined by the neoplasm with the poorest prognosis [5].

There are no specific guidelines regarding the management of synchronous primary gynecological neoplasms. Proper management should be largely individualized taking into consideration several parameters, such as age of patient, disease type, disease stage, disease grade, and extent of the neoplastic invasion [10]. Management modalities include surgical debulking and/or adjuvant radiotherapy and/or adjuvant chemotherapy, as seen clinically appropriate. Patients with advanced stage, higher grade, and poor histological synchronous primary neoplasms should be treated with more aggressive management modalities.
Clear-cell carcinoma (CCC) of the ovary is regarded as a grade III and high-risk neoplasm, irrespective of the disease stage. Also, CCC of the ovary is recognized to be less sensitive to first-line platinum-based chemotherapy [20]. Previous evidence advocated that irinotecan was more effective against primary [21] and recurrent [22] ovarian CCC when compared to other cytotoxic regimens. However, a recent randomized phase III trial of paclitaxel plus carboplatin (PC) versus irinotecan plus cisplatin (CPT-P) in patients with ovarian CCC showed no superiority of CPT-P over the gold standard $\mathrm{PC}$ regimen [23].

Poorly differentiated squamous cell carcinoma of the cervix is regarded as a high-risk factor influencing the likelihood of recurrence. Hence, an adjuvant therapy may be recommended. The administration of concurrent cisplatinbased chemotherapy and radiation therapy (in the form of EBRT or brachytherapy) has been shown to substantially improve progression-free and overall survival for high-risk, early-stage patients with cervical cancer [24].

\section{Conclusion}

Although exceedingly uncommon, the likelihood of triple synchronous primary neoplasms should be considered when evaluating neoplasms of the female genital system. Careful pathological examination of the surgical specimens can substantially aid in recognizing such synchronous neoplasms.

\section{Conflicts of Interest}

The authors declare that they have no conflicts of interest.

\section{References}

[1] Y.-S. Ree, S.-H. Cho, S.-R. Kim, S.-H. Cho, K.-T. Kim, and M.-H. Park, "Synchronous primary endometrial and ovarian cancer with three different histologic patterns: a case report," International Journal of Gynecological Cancer, vol. 13, no. 5, pp. 678-682, 2003.

[2] A. Ayhan, O. T. Yalcin, Z. S. Tuncer, T. Gurgan, and T. Kucukali, "Synchronous primary malignancies of the female genital tract," European Journal of Obstetrics \& Gynecology and Reproductive Biology, vol. 45, no. 1, pp. 63-66, 1992.

[3] S.-Y. Tong, Y.-S. Lee, J.-S. Park, S.-N. Bae, J.-M. Lee, and S.-E. Namkoong, "Clinical analysis of synchronous primary neoplasms of the female reproductive tract," European Journal of Obstetrics Gynecology and Reproductive Biology, vol. 136, no. 1, pp. 78-82, 2008.

[4] E. Takatori, T. Shoji, Y. Miura, S. Takeuchi, N. Uesugi, and T. Sugiyama, "Triple simultaneous primary invasive gynecological malignancies: a case report," Journal of Obstetrics and Gynaecology Research, vol. 40, no. 2, pp. 627-631, 2014.

[5] B. Chiofalo, J. Di Giuseppe, L. Alessandrini et al., "Triple synchronous invasive malignancies of the female genital tract in a patient with a history of leukemia: a case report and review of the literature," Pathology Research and Practice, vol. 212, no. 6, pp. 573-577, 2016.

[6] C. S. Hale, L. Lee, and K. Mittal, "Triple synchronous primary gynecologic carcinomas: a case report and review of the literature," International Journal of Surgical Pathology, vol. 19, no. 4, pp. 552-555, 2011. 
[7] T. Jobo, H. Iwaya, M. Arai, S. Kamikatahira, and H. Kuramoto, "Early triple malignancies of the female genital tract: a case report," International Journal of Clinical Oncology, vol. 2, no. 1, pp. 51-54, 1997.

[8] A. Saglam, G. Bozdag, G. M. Kuzey, T. Kucukali, and A. Ayhan, "Four synchronous female genital malignancies: the ovary, cervix, endometrium and fallopian tube," Archives of Gynecology and Obstetrics, vol. 277, no. 6, pp. 557-562, 2008.

[9] A. Isin Dogan Ekici, T. Kucukali, M. Coskun Salman, and A. Ayhan, "Triple simultaneous primary gynecological malignancies in a 56-year-old patient," International Journal of Gynecological Cancer, vol. 16, no. 5, pp. 1947-1950, 2006.

[10] M. Atasever, B. Yilmaz, G. Dilek, E. Y. Akcay, and S. Kelekci, "Synchronous primary carcinoma in 5 different organs of a female genital tract: an unusual case and review of the literature," International Journal of Gynecological Cancer, vol. 19, no. 4, pp. 802-807, 2009.

[11] V. Phupong, N. Khemapech, and S. Triratanachat, "Triple synchronous primary cervical, endometrial and ovarian cancer with four different histologic patterns," Archives of Gynecology and Obstetrics, vol. 276, no. 6, pp. 655-658, 2007.

[12] H. Zhang and M. Lerwill, "Co-existence of three rare gynecological tumors in a 79-year-old woman," Archives of Gynecology and Obstetrics, vol. 284, no. 3, pp. 695-698, 2011.

[13] T. Pekin, P. Yoruk, R. Yildizhan, B. Yildizhan, and S. Ramadan, "Three synchronized neoplasms of the female genital tract: an extraordinary presentation," Archives of Gynecology and Obstetrics, vol. 276, no. 5, pp. 541-545, 2007.

[14] D. L. Matlock, F. A. Salem, E. H. Charles, and E. W. Savage, "Synchronous multiple primary neoplasms of the upper female genital tract," Gynecologic Oncology, vol. 13, no. 2, pp. 271-277, 1982.

[15] M. Shinohara, Y. Matsuura, S. Baba, and M. Kashimura, "Multiple primary cancers associated with gynecologic malignancies," Nihon Sanka Fujinka Gakkai Zasshi, vol. 42, no. 6, p. 561, 1990.

[16] D. A. Buchler, "Multiple primaries and gynecologic malignancies," American Journal of Obstetrics and Gynecology, vol. 123, no. 4, pp. 376-381, 1975.

[17] R. F. Eisner, R. K. Nieberg, and J. S. Berek, "Synchronous primary neoplasms of the female reproductive tract," Gynecologic Oncology, vol. 33, no. 3, pp. 335-339, 1989.

[18] P. Eifel, M. Hendrickson, J. Ross, S. Ballon, A. Martinez, and R. Kempson, "Simultaneous presentation of carcinoma involving the ovary and the uterine corpus," Cancer, vol. 50, no. 1, pp. 163$170,1982$.

[19] T. M. Ulbright and L. M. Roth, "Metastatic and independent cancers of the endometrium and ovary: a clinicopathologic study of 34 cases," Human Pathology, vol. 16, no. 1, pp. 28-34, 1985.

[20] T. Sugiyama, T. Kamura, J. Kigawa et al., "Clinical characteristics of clear cell carcinoma of the ovary: a distinct histologic type with poor prognosis and resistance to platinum-based chemotherapy," Cancer, vol. 88, no. 11, pp. 2584-2589, 2000.

[21] S. Takakura, M. Saito, K. Ueda et al., "Irinotecan hydrochloride (CPT-11) and cisplatin as first-line chemotherapy after initial surgery for ovarian clear cell adenocarcinoma," International Surgery, vol. 92, no. 4, pp. 202-208, 2007.

[22] T. Sugiyama, M. Yakushiji, T. Nishida et al., "Irinotecan (CPT-11) combined with cisplatin in patients with refractory or recurrent ovarian cancer," Cancer Letters, vol. 128, no. 2, pp. 211-218, 1998.
[23] T. Sugiyama, A. Okamoto, T. Enomoto et al., "Randomized phase III trial of irinotecan plus cisplatin compared with paclitaxel plus carboplatin as first-line chemotherapy for ovarian clear cell carcinoma: JGOG3017/GCIG trial," Journal of Clinical Oncology, vol. 34, no. 24, pp. 2881-2887, 2016.

[24] W. A. Peters III, P. Y. Liu, R. J. Barrett II et al., "Concurrent chemotherapy and pelvic radiation therapy compared with pelvic radiation therapy alone as adjuvant therapy after radical surgery in high-risk early-stage cancer of the cervix," Journal of Clinical Oncology, vol. 18, no. 8, pp. 1606-1613, 2000. 


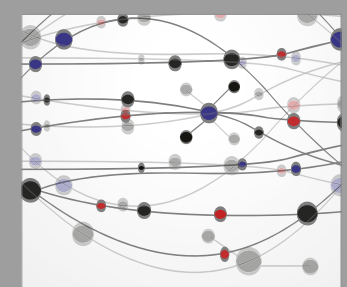

The Scientific World Journal
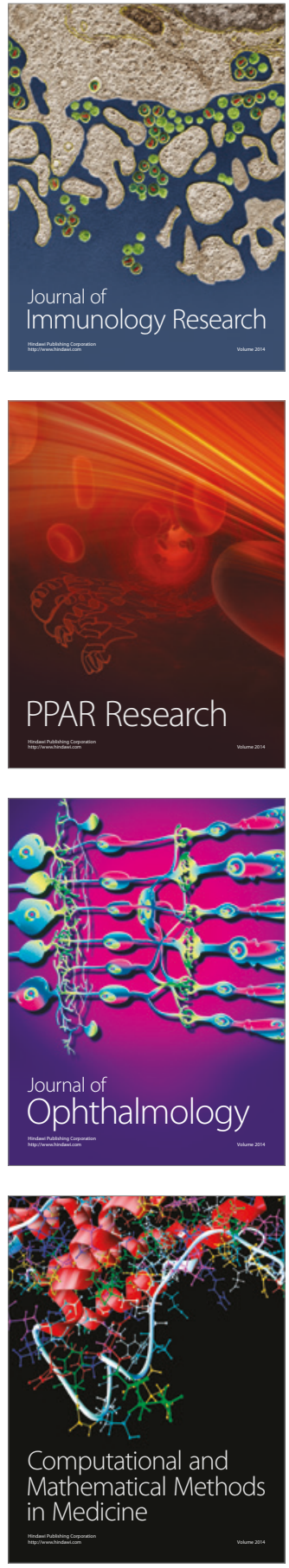

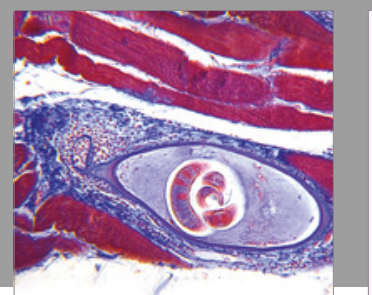

Gastroenterology Research and Practice
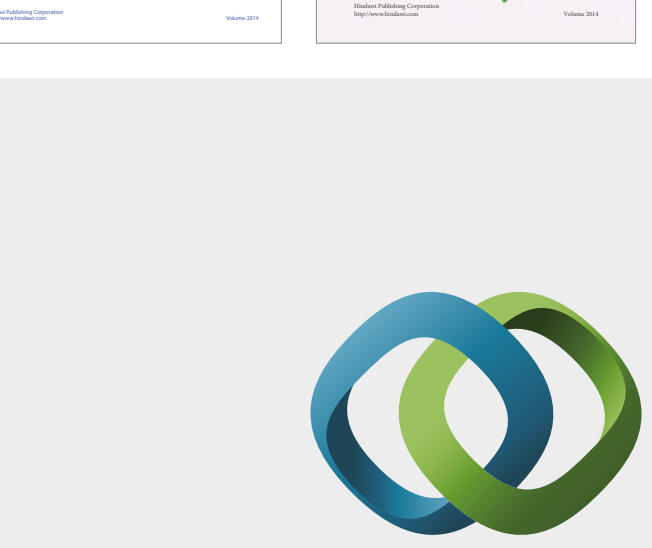

\section{Hindawi}

Submit your manuscripts at

https://www.hindawi.com
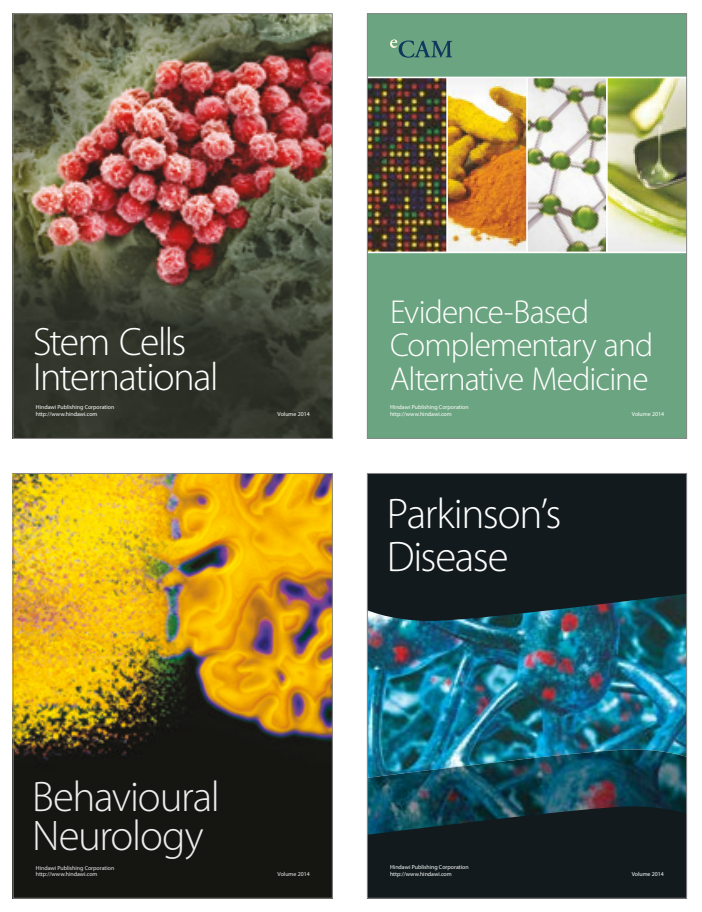
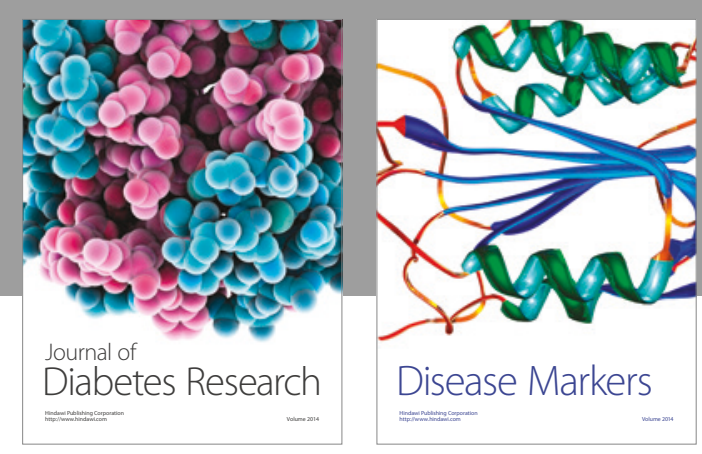

Disease Markers
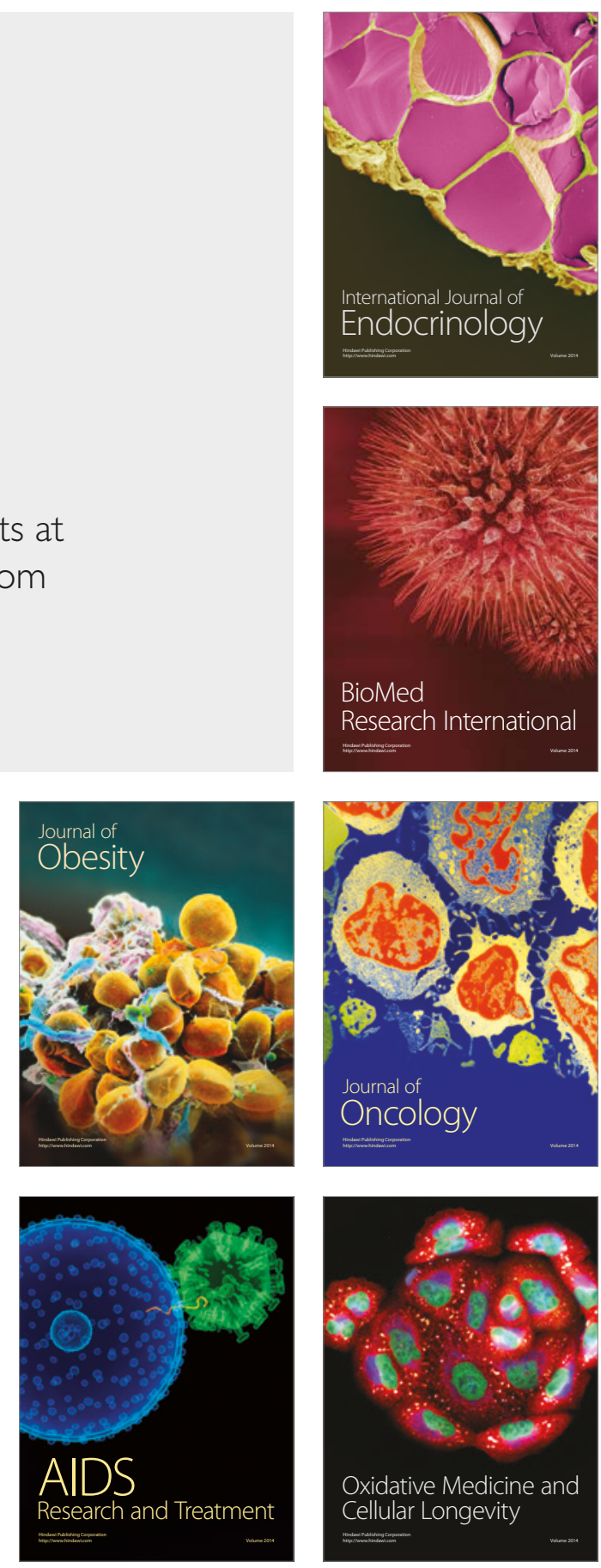